\title{
Continuous Caudal Catheters in Neonatal Population: A Focussed Review
}

\author{
Vrushali Ponde ${ }^{1}$, Kriti Puri ${ }^{2}$, Nandini Dave ${ }^{3}$ \\ ${ }^{1}$ Department of Anaesthesia, Surya Children Anaesthesia Services, Mumbai, Maharashtra, India. \\ ${ }^{2}$ Department of Anaesthesia, Ganga Hospital, Coimbatore, Tamil Nadu, India. \\ ${ }^{3}$ Department of Anaesthesia, NH SRCC Children's Hospital, Mumbai, Maharashtra, India.
}

\section{Abstract}

Caudal epidural block is one of the most commonly administered blocks in paediatric population. Continuous caudal technique offers several advantages like its ability to cater to long duration surgeries, higher thoracic procedures and to deliver extended, titratable post-operative pain relief. Current advances in this technique like use of fluoroscopy, electrical stimulation and Ultrasound to secure continuous caudal catheters facilitate enhanced accuracy and safety and should be adopted wherever feasible. An understanding of local anaesthetic dosages for infusion and their fine tuning is a prerequisite. The potential benefits and risks should be assessed on a case-to-case bases

Keywords: Caudal anaesthesia, Post-operative pain, Neonatal regional anaesthesia

\section{Introduction}

\section{Why this review?}

Given the premise that anaesthesia related neurotoxicity is a real possibility in the neonatal age group, regional anaesthesia (RA) surely offers benefits. Evidently, RA has general anaesthesia sparing effect and its extension into the post-operative period spares opioids, reduces the need for ventilatory support and can aid weaning. In the current datum, we have many options for RA. However, for thoraco abdominal procedures, which are the most common procedures in the neonatal age group, continuous caudal epidural (CCE) technique confers distinct advantages for clear reasons. The pain relief offered by this technique encompasses somatic as well as visceral pain facilitating opioid sparing analgesia. The caudal space is easily accessible in this age group as compared with grown up children and adults wherein with growing age spongy gelatinous material is replaced by dense fibrous tissue. In fact, until one year of age this connective tissue remains relatively soft. $[1,2]$ Moreover [3], in neonates, due to absence of secondary curvatures of spine, especially the lordotic curve in the lumbar region, catheters can be advanced rostrally to long distances. The efficacy of the CCE is determined by the congruency of the catheter tip to the surgical incision. Various techniques have been implemented to detect the catheter tip to ensure optimal analgesia. However, studies have also shown migrations of the caudal epidural catheter defeating the very purpose of their placement. $[4,5]$ On the other side of the spectrum, substantial progress has been made in our understanding of this procedure. The catheter tip placement can be confirmed by various modalities such as radiographic imaging, neurostimulation, and application of ultrasonography (US). The latter allows real time visualization of catheter trajectory, and its tip while securing it, moreover, also aids in excluding any anatomical abnormality and obviates the risk of radiographic exposure. [6] Various alternative RA techniques are available such as erector spine, and paravertebral blocks. However, the bilateral coverage CCE offers especially to laparotomies, and the easy accessibility of caudal epidural space in this neonatal group remain some of its most positive points. Furthermore, CCE can be performed without additional modalities using the traditional "blind technique" and can be fine tuned further if US and above-named modalities are available.

Considering the scope and play of this subject, we aim to review the literature, examining the historical perspective, recent data, current practices, and recommendations pertaining to CCE technique in neonates specifically. This review also offers technical aspects of CCE and current recommendations. Thus, the readers shall find comprehensive information on the know how's of this technique.

Address of Correspondence: Dr. Vrushali Ponde, Consultant Paediatric Anaesthesiologist, Surya Children Anaesthesia Services,

Mumbai, Maharashtra, India. E-mail: vrushaliponde@yahoo.co.in

Submitted: 3 March 2021; Reviewed: 10 April 2021; Accepted: 20 April 2021; Published: 10 July 2021

\section{DOI: 10.13107/ijra.2021.v02i02.040 | www.ijrajournal.com |}

This is an Open Access article distributed under the terms of the Creative Commons Attribution Non-Commercial-Share Alike 4.0 License

(http://creativecommons.org/licenses/by-nc-sa/4.0) which allows others to remix, tweak, and build upon the work non-commercially as long as appropriate credit is given and the new creation are licensed under the identical terms. 


\section{Historical perspective}

The sacral hiatus has been used as a portal to introduce catheters into the neuraxial conduit since decades and has endured the test of time. Ruston described anaesthetic technique combining CCE and GA in early 1950's. [7] In 1988, Bosenberg et al introduced continuous thoracic epidural anaesthesia via caudal route in infants and children. [2] Furthermore, Ponde et al reported accurate advancement of caudal catheter upwards to the desired level under US guidance. [6] Recently, Bachman et al [7] have described thoracic caudal epidural localization using ultrasound guidance. In a nut shell, CCE has endured the test of time and still has a definite scope in perioperative pain management in neonates.

\section{Methodology}

A comprehensive literature search was conducted in December 2020 in PubMed and Google Scholar to retrieve articles pertaining to continuous caudal blocks in the last 20 years. The keywords used were 'caudal anaesthesia, epidural anaesthesia, acute pain services, neonates, paediatrics, ultrasound, complications, and safety'.

To systematically cover every aspect and recent trends of CCE we divided the review under the following headings.

Indications

Advantages

Contraindications

Techniques

LA and infusion dosages

Complications

Management of CCE in the postoperative period

Summary

\section{Indications}

CCE is beneficial in Neonatal laparotomies for malrotations, perforations, necrotizing enterocolitis etc. Also, thoracotomies, tracheoesophageal fistula (TEF), congenital lobar emphysema and congenital diaphragmatic hernia $(\mathrm{CDH})$ repair. It may be used as a sole anaesthetic in awake preterm babies with a post-conceptional age (PCA) of $<60$ weeks wherein postoperative apnoea is a major risk. Several authors have described its successful use for infraumbilical and for major abdominal procedures. $[8,9]$ It is indispensable in case spontaneous respiration is required intraoperatively, like in cases of congenital lobar emphysema and TEF. [10] CCE has also been used successfully for treatment of arterial thrombosis of the lower extremities in neonates. [11]

\section{Advantages of CCE}

1. The innate safety and easy accessibility of a trans caudal catheter approach makes it very advantageous and averts many risks. Direct lumbar or thoracic epidural placement should be reserved for experts because the distance from skin to epidural space in neonates may be as little as $5 \mathrm{~mm}$, increasing the possibility of inadvertent dural puncture and/or spinal cord injury. [12]

2. The catheter can cover a wider dermatomal range overcoming the limitation of its corresponding single shot technique. It can be manipulated to position the catheter tip congruent to the surgical incision. It behaves like a sitespecific epidural once it is optimally placed, restricting the dose and volume to be administered. This reduces the probability of local anaesthetic systemic toxicity (LAST).

3. The ability to continuously infuse and titrate local anaesthetics (LA) is particularly helpful in prolonged procedures.

4. Intraoperatively it causes less hemodynamic alteration.

5. In the presence of a well acting block, spontaneous respiration can be instituted if needed.

6. Continuous catheter technique offers excellent analgesia avoiding systemic opioids which enables early extubation and improved outcomes.

This fact is substantiated by a study performed in children undergoing fundoplication [13] and patent ductus arteriosus ligation, with caudal catheter insitu. [14]

7. CCE aids peristalsis and has a positive immunological and metabolic response. [15]

\section{Contraindications}

Coagulopathy, systemic infections, allergy to LA, localized skin infection at puncture site, skeletal spinal deformity and non-consenting parents are few contraindications to caudal catheter technique. It is noteworthy to mention that due to immaturity in hepatic function, coagulation tests in neonates may yield abnormal results even in the presence of clinically intact function. In case of spinal deformities, an MRI scan may be prudent to perform a risk-benefit analysis.

\section{Technique \\ Equipment}

$19 \mathrm{G}$ or $20 \mathrm{G}$ Tuohy needle with their respective catheters, Loss of resistance (LOR) syringe, normal saline and material for skin preparation.

\section{Anatomy and Positioning}

The caudal space is accessed via conventional method of identifying the sacral hiatus. The patient is kept in left or right lateral decubitus. In neonates the anatomical 


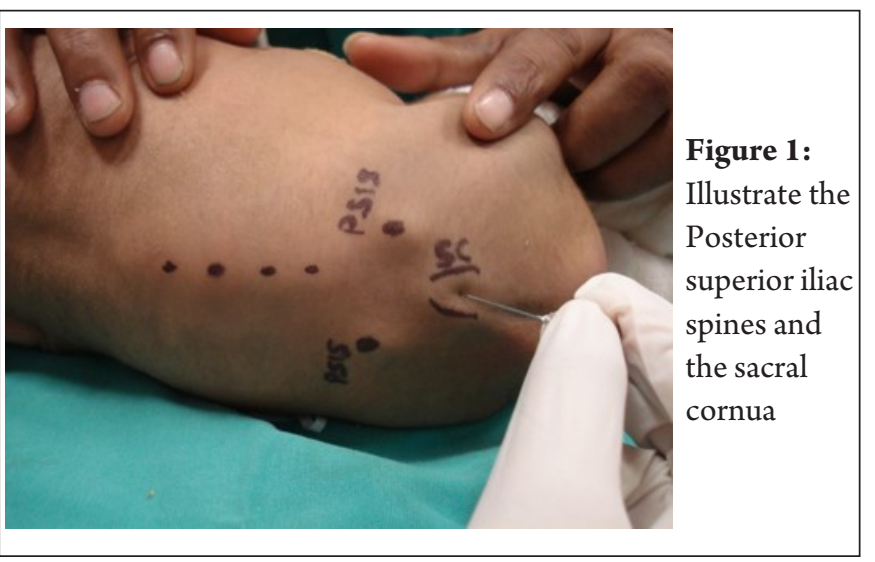

landmarks are very clear. The posterior superior iliac spines are seen as well as easily palpated. An imaginary line connecting them marks one side of an equilateral triangle. Two bony prominences of the sacral cornua are identified and in between is located the sacral hiatus. (Figure 1)

The distance between the sacral hiatus and the surgically congruent vertebral level is estimated in centimeters by measuring tape if possible. This determines the length of the catheter to be inserted inside the caudal epidural space.

\section{Technique}

\section{For the landmark guided technique.}

Under aseptic technique, the Touhy needle is inserted at an angle of approximately $30-45$ degrees from the skin surface and advanced till a gentle 'give' or pop is felt. This signifies piercing of sacrococcygeal ligament and entry into caudal epidural space. To aid catheter insertion 0.5 to $1 \mathrm{ml}$ of normal saline can be injected. This confirms the correct placement of the needle, as denoted by absence of subcutaneous swelling and also aids catheter trajectory. The catheter is threaded and advanced cephalad to a predetermined length depending on the surgical incision. Usually, the catheter threading is very smooth. In case of resistance, a small bolus of saline or LA does the trick. Once the required length is lodged in the epidural space, the needle is withdrawn outward, while carefully inserting the catheter inside. Should this manoeuvre result in excessive length of catheter insertion, it can be withdrawn to the required mark.

Note: Patients with urogenital anomaly may have a high incidence of lower spinal dysraphism [16] and a preprocedure US screening is recommended to assess the riskbenefit.

\section{Procedure for the ultrasound guided real time technique}

A high frequency transducer is prepared with a camera cover and glove technique or any other technique that suits the local work culture as long strict asepsis is followed. After identifying the lumbosacral junction in longitudinal sagittal view, scan the vertebrae cephalad to the surgically congruent level. One anesthesiologist performs the continuous caudalepidural block while the second anesthesiologist follows the needle entry into the caudal space and the catheter trajectory. Alternatively, both can be done by a single anaesthesiologist by intermittent scanning at appropriate time points. [3]

It has been documented that the accuracy is improved by ultrasound guidance. [6] Catheter fixation, dosages of LA, and postoperative care remains the same for both techniques.

\section{Methods of confirmation}

Various methods to confirm optimal catheter placement include radiological methods such as epidurograms or fluoroscopy, electrical nerve stimulation and US. $[17,18]$ Fluoroscopic or US guidance improves accuracy of trans caudal technique and obviates the need for larger doses of LA or addition of opioids/additives to compensate for a sub optimally placed epidural catheter.

\section{Test dose}

A test dose of LA has been described to detect intravascular placement. Some studies advocate a higher than usual test dose of $0.75 \mathrm{mcg} / \mathrm{kg}$ epinephrine. Premedication with atropine may prove beneficial. Patients should be observed for at least 90 seconds after test dose. [19] Criteria for a positive test dose include increase $\geq 25 \%$ of T-wave amplitude, heart rate increase $\geq 10$ beats per min and $\geq 15$ $\mathrm{mmHg}$ increase in systolic blood pressure. Due to the small test dose volume in neonates, it is important to flush the catheter to determine that the test dose was essentially delivered. Experts advise that each top-up administered via catheter should be regarded as a test dose and delivered in small aliquots.

\section{LADosage}

Once the CCE is insitu, an initial bolus of $0.3-0.5 \mathrm{ml} / \mathrm{kg}$ is given, table 1 illustrates the drugs and dosages.

Dose should be modified with $20-30 \%$ reduction in weightbased dosing due to decreased hepatic clearance and alpha1-glycoprotein concentration causing higher free fraction of drug in neonates, especially premature babies.

\section{Additives}

Addition of adjuvants to CCE infusion in neonates is very sparingly described. This is understandable, because the duration of analgesia can be prolonged by the LA infusion 


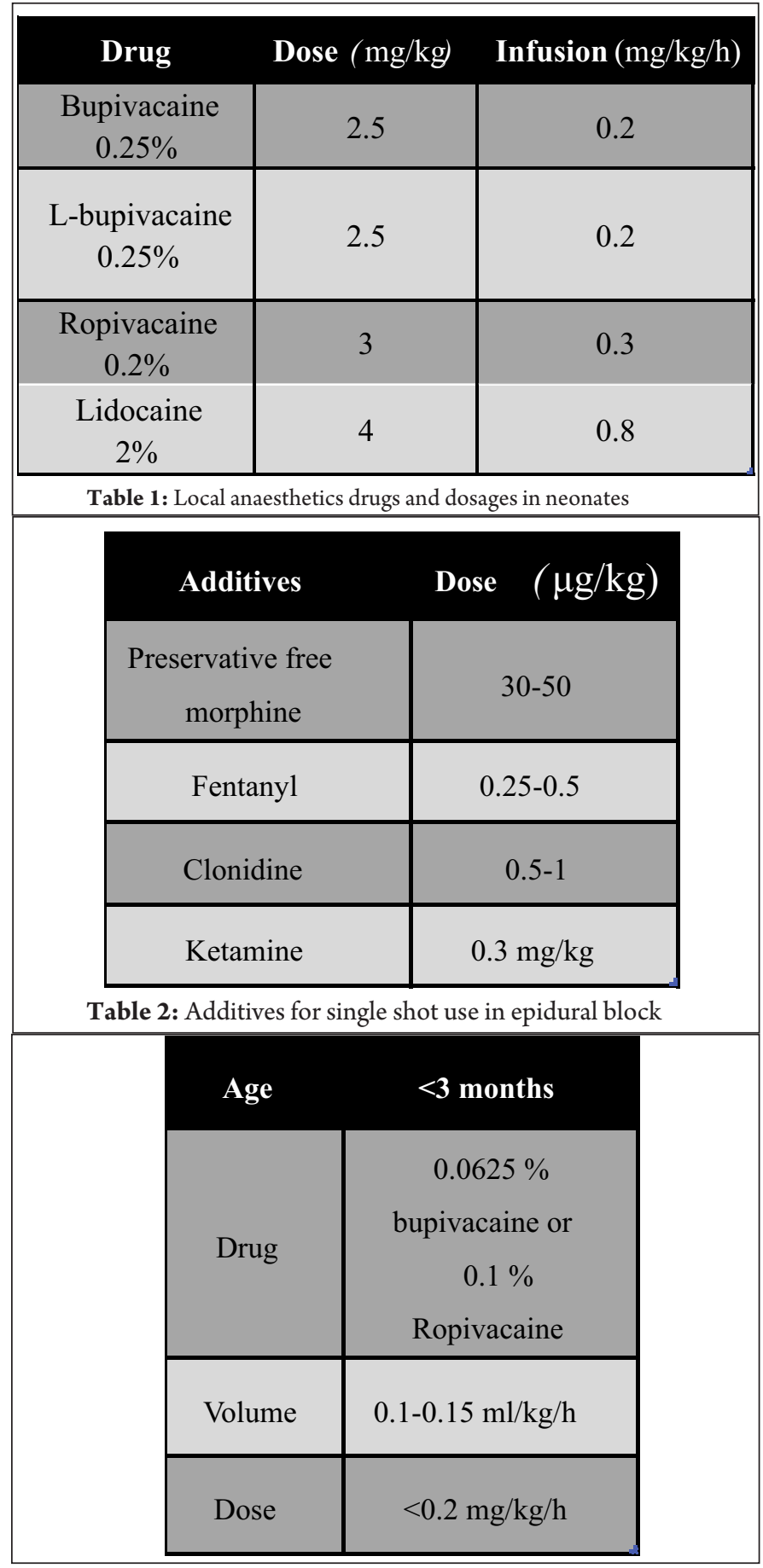

itself. This strategy prevents the side effects that addition of adjuvants may cause without really adding any substantial benefits.

However, preservative-free morphine and clonidine are registered drugs for epidural use. The recommendations issued by the European/American Society of Regional Anaesthesia endorse the use of alpha-2 agonists (clonidine, dexmedetomidine), preservative-free morphine, and ketamine as adjuvants in caudal blocks. [20]

Clonidine (median concentration $0.2 \mu \mathrm{g} / \mathrm{mL}$ ) infused at a median rate of $0.72 \mathrm{~mL} / \mathrm{kg} /$ hour along with chlorprocaine is described by Gibbs et al. [21]

\section{Infusion techniques}

Infusion pumps can ensure continuous administration of epidural LA. Caution and vigilance needs to be exercised while using continuous infusion techniques. Drug accumulation and cumulative toxicity can be serious complications. Generally, syringe infusion pumps are used in neonates. Elastomeric pumps may not deliver the very small volumes which are sufficient for this age group. In case the syringe pump is used; it has to be labelled accurately, mentioning "NOT FOR IV USE" in bold letters.

\section{Complications}

Paediatric regional anaesthesia per se has a wide safety margin. However neonatal CCE needs special consideration. Giaufre et al [22] evaluated 15013 central blocks in children, wherein they reported a complication rate of $0 \%$ in term and preterm neonates. This data may be misleading as only 29 neuraxial catheters were placed in this age group. PRAN (Paediatric regional anaesthesia network) data suggests that in 730 caudally threaded epidural catheters in paediatric patients, no permanent neurologic deficits, or epidural hematomas were noted. [23]

\section{- Dural puncture}

In neonates and small children, the dural sac can be accessed within millimetres of sacral hiatus owing to thin skin and negligible adipose tissue. Thus, careful manoeuvring of needle is warranted. Negative aspiration if not confirmed can cause inadvertent intrathecal spread of LA leading to potentially catastrophic effects. There is insufficient data to highlight consequences of wet tap following dural puncture in neonates.

\section{- Trauma}

Spinal cord injury has been reported in infants [24, 25] undergoing lumbar epidural catheter placement. Theoretically, caudal technique possesses minimal risk of neural injury since the spinal cord terminates higher above the needle introduction site. Nonetheless, risk of cord, root or nerve injury from advancing the catheter cranially is a remote possibility. From a practical point of view, in case resistance is encountered, catheter advancement must be stopped immediately.

\section{- Blood tap}

The authors have noticed increased incidence of bloody tap in patients having a distended abdomen. A potential explanation could be increased abdominal pressure being transmitted to epidural veins. However, we did not find adequate data to validate our observation. 


\section{- LAST}

LAST is rare, however can be fatal in infants and neonates. The existing case reports are predominantly in newborns and infants. It can be prevented by strictly adhering to maximal allowable LA doses, use of a test dose and incremental injections in aliquots. One should be mindful of the fact that general anaesthesia can mask the presentation of LAST. The manifestation of central nervous system toxicity, in the form of seizures, (if muscle relaxant is not given), calls for airway control and oxygenation, control of seizures with midazolam and thiopentone, arterial blood gases and, if possible, druglevel sampling.

- Cardiovascular toxicity is treated with $1.5 \mathrm{ml} / \mathrm{kg}$ to 2 $\mathrm{ml} / \mathrm{kg}$ of $20 \%$ lipid emulsion bolus intravenously over 1 $\mathrm{min}$. The bolus can be repeated once or twice if required. The continuous infusion recommended dose is of 0.25 $\mathrm{ml} / \mathrm{kg} / \mathrm{min}$ (upper limit of $10 \mathrm{ml} / \mathrm{kg}$ lipid emulsion over the first $30 \mathrm{~min}$ ). Supportive treatment includes fluids and vasopressors.

\section{- Malposition}

CCE catheters may inadvertently enter the intravascular, intrathecal or even presacral space. [1]

\section{Adverse effects of additives}

Additives should be used with caution in neonates. [26] Epidural narcotics, and clonidine, also have been used in neonates and small infants. [27] strict monitoring for respiratory depression should be pursued. [28 ] Dose of additives should be reduced for neonates. In view of reports of apnoea following caudal clonidine, [29] its use in neonates is controversial.

\section{- Leak from epidural site}

This is an expected sequel more common in low birth neonates due to proximity between skin and epidural space. The larger diameter of the Tuohy needle and the smaller epidural catheter, along with the small epidural space compounds the leak. This may not hinder the effectiveness of the block, but constant soakage of LA may lead to inadvertent catheter removal. [30]

\section{Infection}

There is a possibility of bacterial colonization leading to epidural abscess/infection. Studies comparing the bacterial colonization and infection rates of caudal and lumbar epidural catheters [31-34] reported significantly faster colonization of caudal catheters due potential contamination from stools. Preventive measures such as

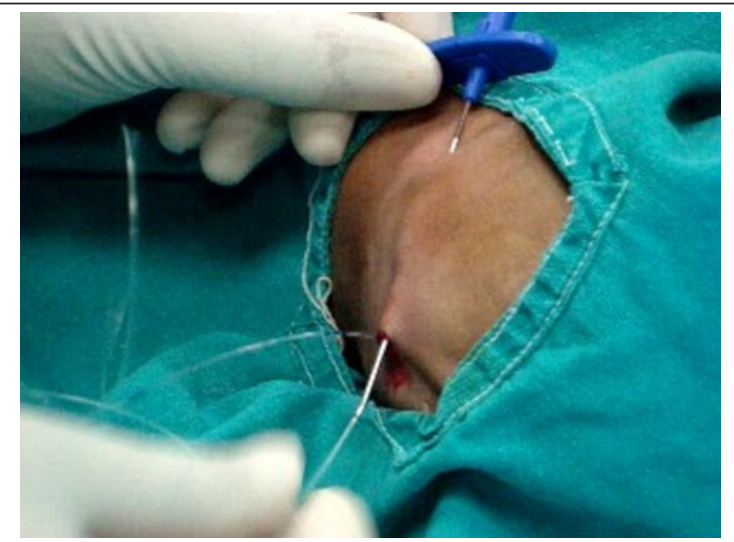

Figure 2: Illustrates subcutaneous tunneling of caudal epidural catheter

cutaneous tunnelling (Figure 2) of the catheter can avoid contamination and soiling of caudal catheters. In 2004 Bubeck et al, conducted a study on 409 children corroborating the above recommendation. They found a bacterial colonization rate of $29 \%$ in non-tunnelled caudal catheters, $11 \%$ in tunnelled caudal catheters, and $9 \%$ in nontunnelled lumbar catheters. [35] Cutaneous antisepsis with chlorhexidine proved superior to povidone iodine in averting bacterial colonization during lumbar epidural placement and this data may be extrapolated to caudal catheter placement as well. [36]

\section{Management of neonates with caudal catheters}

A caudal catheter in neonates necessitates perioperative monitoring of the patient by anaesthesiologist and/or trained personnel. Neonates with indwelling catheters need to be observed closely in intensive care units. Assessment of neonatal pain and discomfort is a challenging task. Several scores and scales have been devised considering behavioural and physiologic parameters. [37] Systematic documentation of pain scores at least on hourly basis and clinical assessment of patients should be undertaken. [38]

\section{Monitoring the neonate with a caudal catheter}

In the authors' institution, neonatal epidural catheters are managed in the postoperative high dependency unit. Round-the-clock availability of a dedicated paediatric pain service is imperative for the successful and safe management of epidural analgesia in neonates. Pain management staff should comprise of personnel well versed with management of paediatric epidurals including ability to detect and treat any untoward event. Strict supervision with continuous electronic monitoring of vital signs including heart rate, blood pressure, oxygen saturation and respiratory monitoring is advocated. Local anaesthetic infusion should be started at lower rates and titrated to desired effect. 


\section{Removal of catheter}

On an average, catheters in neonates are removed after 48 hours. Removal essentially is very easy. However, Joselyn A [39] et have described resistance while removal. With repositioning and various other maneuvers, the catheter was removed with some difficulty. On examination of the catheter, a complete knot was noted.

\section{Summary}

With all the hoopla about GA and neurotoxicity, we must finally take a pragmatic approach. Amidst the plethora RA techniques available CCE has an unquestionable role. While there is no doubt about their benefits, we must use drugs with proven efficacy and widest safety margin especially in neonatal population. Expertise and suitable equipment have no replacement. Adding adjuvants in CCE calls for little logic as the LA infusion itself extends the blocks. In case one chooses to, we must examine the evidence closely to ensure that safety is not compromised at any cost.

Declaration of patient consent: The authors certify that they have obtained all appropriate patient consent forms. In the form, the patient has given his consent for his images and other clinical information to be reported in the Journal. The patient understands that his name and initials will not be published, and due efforts will be made to conceal his identity, but anonymity cannot be guaranteed.

Conflict of interest: Nil Source of support: None

\section{References}

1. Wiegele M, Marhofer P, Lönnqvist PA. Caudal epidural blocks in paediatric patients: a review and practical considerations. Br J Anaesth. 2019Apr;122:509-517.

2. Bosenberg AT, Bland BAR, Schulte-Steinberg O, Downing JW. Thoracic epidural anaesthesia via the caudal route in infants. Anaesthesiology 1988;69:265-9.

3. Kil HK. Caudal and epidural blocks in infants and small children: historical perspective and ultrasound-guided approaches. Korean J Anesthesiol.2018;71:430-439.

4. Simpao A, GálvezJ, Wartman E, England W, Wu L, Rehman Met al. The Migration of Caudally Threaded Thoracic Epidural Catheters in Neonates and Infants. Anesthesia \& Analgesia. 2019;129:477-481.

5. Baidya D, Pawar D, Dehran M, Gupta A. Advancement of epidural catheter from lumbar to thoracic space in children: Comparison between $18 \mathrm{G}$ and $23 \mathrm{G}$ catheters. Journal of Anaesthesiology Clinical Pharmacology.2012;28:21.

6. Ponde VC, Bedekar VV, Desai AP, Puranik KA. Does ultrasound guidance add accuracy to continuous caudal epidural catheter placements in neonates and infants? Paediatr Anaesth. 2017 Oct;27:1010-1014.

7. Bachman SA, Taenzer AH. Thoracic caudal epidural catheter localization using ultrasound guidance. Paediatr Anaesth. 2020 Feb;30:194-195.

8. Daftary S, R Jagtap. Caudal epidural as a sole anaesthetic in preterm, former preterm and high-risk infants. Indian J. Anaesth. 2005;49:195198.

9. Uguralp S, Mutus M, Koroglu A, Gurbuz N, Koltuksuz U, Demircan M. Regional anesthesia is a good alternative to general anesthesia in pediatric surgery: Experience in 1,554 children.J Pediatr Surg. 2002 ;37:610-3.

10. Raghavendran S, Diwan R, Shah T, Vas L. Continuous caudal epidural analgesia for congenital lobar emphysema: a report of three cases. Anaesth Analg. 2001Aug;93:348-50.

11. Carolis MPD, Bersani I, Piersigili F et al. Peripheral nerve blockade and neonatal limb ischemia: Our experience and literature review. Clinical and applied thrombosis/ haemostasis. 2014Jan:55-60.
12. Luz G, Ladner E, Innerhofer P, Deusch E. Accidents following extradural analgesia in children. The results of a retrospective study. Paediatr Anaesth 1995;5:273.

13. McNeely J, Faber N, Rusy L, Hoffman G. Epidural analgesia improves outcome following paediatric fundoplication: a retrospective analysis. Reg Anaesth 1997; 22: 16-23.

14. Lin, Y.C, Sentivany Collins S.K, Peterson K.L, Boltz M.G and Krane E.J. Outcomes after single injection caudal epidural versus continuous infusion epidural via caudal approach for postoperative analgesia in infants and children undergoing patent ductus ligation. Paediatr Anaesth $1999 ; 9: 134-143$.

15. Bosenberg A. Benefits of regional anesthesia in children. Paediatr Anaesth. 2012 Jan;22:10-8.

16. Koo BN, Hong JY, Song HT, Kim JM, Kil HK. Ultrasonography reveals a high prevalence of lower spinal dysraphismin childrenwith urogenital anomalies. Acta Anaesthesiol Scand. 2012;56:624-8.

17. Tsui BC, Seal R, Koller J. Thoracic epidural catheter placement via the caudal approach in infants by using electrocardio- graphic guidance. Anesth Analg. 2002;95:326-330.

18. Tsui BC, Seal R, Koller J, Entwistle L, Haugen R, Kearney R. Thoracic epidural analgesia via the caudal approach in pediat- ric patients undergoing fundoplication using nerve stimulation guidance. Anesth Analg. 2001;93:1152-1155.

19. Tobias J.D. Caudal epidural block : Review of test dosing and recognition of systemic injection in children. Anaesth Analg. 2001;93:1156-61.

20. Suresh S, Ecoffey C, Bosenberg A, et al. The European society of regional anaesthesia and pain therapy/American society of regional anesthesia and pain medicine recommendations on local anesthetics and adjuvants dosage in pediatric regional anesthesia. Reg Anesth Pain Med 2018; 43:211-6.

21. Gibbs A, Kim SS, Heydinger G, Veneziano G, Tobias J. Postoperative Analgesia in Neonates and Infants Using Epidural Chloroprocaine and Clonidine.J Pain Res. 2020;13:2749-2755. 
22. GiaufreÂ E, Dalens B, Gombert A. Epidemiology and morbidity of regional anaesthesia in children: a one-year prospective survey of the French-Language Society of Paediatric Anaesthesiologists. Anaesth Analg 1996; 83:904912.

23. Suresh, Santhanam MD*; Long, Justin MD*; Birmingham, Patrick K. $\mathrm{MD}^{*}$; De Oliveira, Gildasio S. Jr MD, MSCI $†$ Are Caudal Blocks for Pain Control Safe in Children? An Analysis of 18,650 Caudal Blocks from the Pediatric Regional Anesthesia Network (PRAN) Database, Anaesth Analg. 2015<120 151-156.

24. Breschan C, Krumpholz R, Jost R, Likar R. Intraspinal haematoma following lumbar epidural anaesthesia in a neonate. Paediatr Anaesth $2001 ; 11: 105-108$.

25. Apthorp M, Challands J, Visram A. A survey of the usage of caudal catheters amongst paediatric anaesthetists practising in the UK [Abstract]. Paediatr Anaesth 2000; 10:692.

26. Walker SM, Yaksh TL. Neuraxial analgesia in neonates and infants: a review of clinical and preclinical strategies for the development of safety and efficacy data. Anesth Analg. 2012;115:638-662.

27. Lejus C, Surbled M, Schwoerer D, et al. Postoperative epidural analgesia with bupivacaine and fentanyl: hourly pain assessment in 348 paediatric cases. Paediatr Anaesth 2001; 11:327-332.

28. Breschan C, Krumpholz R, Likar R, et al. Can a dose of $2 \mathrm{mg} \mathrm{kg71}$ caudal clonidine cause respiratory depression in neonates? Paediatr Anaesth 1999; 9:81-83.

29. Bouchut JC, Dubois R, Godard J. Clonidine in preterm-infant caudal anesthesia may be responsible for postoperative apnea. Reg Anesth Pain Med 2001; 26:83-85.

30. Wood CE, Goresky GV, Klassen KA, et al. Complications of continuous epidural infusions for postoperative analgesia in children. Can JAnaesth 1994; 41:613-620.
31. Boos K, Beushausen T, Ohrdorf W. Peridural catheter for postoperative long-term analgesia in children. AnaÈ sthesiol Intensivmed Notfallmed Schmerzther 1996; 31:362-367.

32. Aram L, Krane EJ, Kozloski LJ, Yaster M. Tunneled epidural catheters for prolonged analgesia in pediatric patients. Anesth Analg 2001; 92:1432-1438.

33. Fujinaka W, Hinomoto N, Saeki S, et al. Decreased risk of catheter infection in infants and children using subcutaneous tunneling for continuous caudal anesthesia. Acta Med Okayama 2001; 55:283-287.

34. Vas L, Naik V, Patil B, Sanzgiri S. Tunnelling of caudal epidural catheters in infants. Paediatr Anaesth 2000; 10:149-154.

35. Bubeck J, Boos K, Krause H, Thies K. Subcutaneous Tunneling of Caudal Catheters Reduces the Rate of Bacterial Colonization to That of Lumbar Epidural Catheters. Anesthesia \& Analgesia. 2004;99:68 -693.

36. Kinirons B, Mimoz O, Lafendi L, et al. Chlorhexidine versus povidone iodine in preventing colonization of continuous epidural catheters in children: a randomized, controlled trial. Anaesthesiology 2001; 94:239244.

37. BuÈttner W, Finke W. Analysis of behavioural and physiological parameters for the assessment of postoperative analgesic demand in newborns, infants and young children: a comprehensive report on seven consecutive studies. Paediatr Anaesth 2000; 10:303-318.

38. Lejus C, Surbled M, Schwoerer D, et al. Postoperative epidural analgesia with bupivacaine and fentanyl: hourly pain assessment in 348 paediatric cases. Paediatr Anaesth 2001; 11:327-332.

39. Joselyn A,Bhalla T, Schloss B, Martin D,Tobias J.A case report of a retained and knotted caudal epidural catheter.Saudi J Anaesth $2014 ; 8: 424$.

How to cite this article: Ponde V, Puri K, Dave N | Continuous Caudal Catheters in Neonatal Population: A Focussed Review | International Journal of Regional Anaesthesia | July-December 2021; 2(2): 124-130. 